\title{
An Iterative Interaction-Design Method for Multi-Modal Robot Communication
}

\author{
Elie Saad $^{1}$, Joost Broekens $^{2}$ and Mark A. Neerincx ${ }^{1,3}$
}

\begin{abstract}
The design space of human-robot interaction is large and multi-dimensional. A sound design requires a systematic theory-driven exploration, specification and refinement of design variables. There is a need for a practical method and tool to iteratively specify the content of the dialogue (e.g., speech acts) with the accompanying expressive behavior (e.g., gesture openness) as prescribed by social science theory, e.g., task- and person-oriented communication. This paper presents an iterative interaction-design (ID) method for multi-modal robot communication. Following the $I D$-method, a designer first creates his/her "own" individual design and, subsequently, provides an iteration to the evolving iterative design. To support the design method, we developed an $I D$-tool (available for download). The tool support entails (a) selecting the theory-based communication style; (b) creating and linking the dialogue act components for the concerning use case; and (c) setting the associated expression parameters.

We conducted a study with Industrial Design students $(N=$ 13) who followed the $I D$-method and used our tool to design person- and task-oriented communications for a reception robot. Our method produced distinctive task- and person-oriented dialogue styles, i.e., provided the predicted theory-based multimodal communicative behaviors. The task-oriented style showed a more formal, shorter and less chatty communication. Overall, there was a rather smooth design convergence process, in which the individual designs were harmonized into the iterative design. For the selected design problem, the $I D$-tool had a satisfactory usability. Next steps include validation of the communication styles in an empirical study and, subsequently, identification of reusable design patterns.
\end{abstract}

Index Terms-Human-Robot Interaction; Multi-modal Communication; Iterative Design; Communication Styles; Expressive Behaviors; Interaction Design; Reception Robot.

\section{INTRODUCTION}

The introduction of social robots in public spaces (e.g., airports and hospitals) to interact with and assist human visitors, has raised several challenges related to human-robot engagement and communication. One of the main challenges is to harmonize the engagement and communicative approach to the individual and social contextual factors. Appropriate use of communication styles and being socially competent, i.e., appropriately initiating, maintaining and closing an interaction (e.g., [1], [2]), are required to attune engagement to the goal, the situation and the person.

Visitors repeatedly arrive at the reception of different organizations, including meeting centers and hospitals, and

\footnotetext{
${ }^{1}$ Faculty of Electrical Engineering, Mathematics and Computer Science, Department of Intelligent Systems, Delft University of Technology, Delft, The Netherlands. E.Saadetudelft.nl

2 The Leiden Institute of Advanced Computer Science, Leiden University, Leiden, The Netherlands. Joost.Broekens@gmail.com

3 The Netherlands Organization for Applied Scientific Research (TNO), Soesterberg, The Netherlands. Mark. Neerincx@tno.nl
}

social robots can help in receiving and guiding them to the appropriate service. This will somewhat free (human) staff from basic and repetitive tasks such as welcoming visitors, to spend more time on providing them with more meaningful guidance. In this study, we focus on the reception in healthcare which (1) provides a "rich" test environment for studying relevant human-robot interaction (HRI) aspects, i.e., fit for research; and (2) entails relatively high potential benefits for robot deployment, i.e., fit for application. In particular, the interest lies in the high workload of receptionists who have to deal with both administrative and visitor support tasks (e.g., [3]); the rather continuous attendance of visitors who have to be accommodated appropriately (e.g., [4]); and the diversity of the visitors' state which affects the choice of the communicative behavior (e.g., [5]).

In a healthcare establishment, receptionists are usually the first point of contact with visitors (e.g., [6]) who depend on them to obtain access to care, such as speaking to a doctor or checking-in for an appointment. The interaction between healthcare receptionists (HCRs) and visitors consists mainly of a technical exchange of information, i.e., task related, and may also include personal exchange of information, i.e., person related (e.g., [4]). Studies have shown that the behavior of healthcare staff (i.e., their caring approach) impacts the visitors' acceptability of the provided service and influences their continuity of care (e.g., [7], [3]). Therefore, introducing new technologies into healthcare - in particular technology that requires communication with visitors such as social robots - needs to be carefully examined and designed to facilitate and positively affect the patient's journey.

The design space of HRI is large and multi-dimensional. A sound design requires a systematic theory-driven exploration, specification and refinement of design variables. There is a need for a practical method and tool to iteratively specify the interaction content (e.g., speech acts) with the accompanying expressive behavior (e.g., gesture openness and voice pitch). Our research question is three-fold: (1) How can we support the proposed design refinement process, (2) does such support bring about the desired theory-based different designs, and (3) how does the refinement process evolve over different designers?

First, we developed a design support tool for (a) selecting the theory-based communication style (i.e., task- and person-oriented); (b) creating and linking the dialogue act components for the use case; and (c) setting the associated expression parameters. Second, we linked this tool to the 
humanoid robot Pepper ${ }^{1}$ (Fig. 1), which expresses all (interim) design specifications (so, allowing for a systematic exploration of the design space). Third, we conducted a study with Industrial Design students $(N=13)$, as in [8]. Using the tool, the designers followed a process that consists of both the creation of an individual design and a contribution to an iterative design: They had to provide their own design of person- and task-oriented communications for a reception robot acting as an HCR assistant and, subsequently, refine the design that the previous participant had produced. This way, we could investigate how subsequent individual designs relate to the evolving iterations.

The remaining of this paper is organized as follows. In Section III we review related work. In Section III we discuss modeling the communication styles. In Section IV we present our hypotheses. In Sections V] and VI we discuss the methods and analyze the results. In Section VII we discuss the findings and conclude the paper.

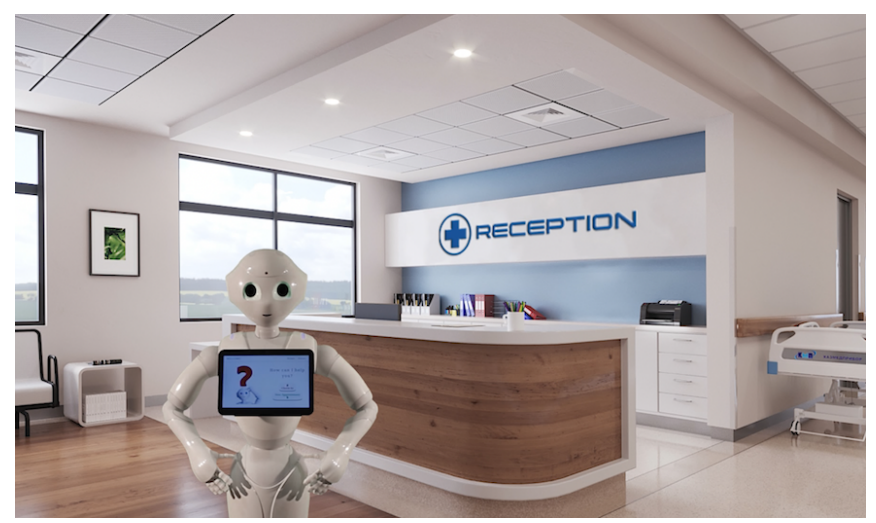

Fig. 1. The Pepper robot acting as a receptionist assistant (the 3D rendering of the background image is courtesy of Artistic Visions, LLC, https: l/artisticvisions.com).

\section{RELATED WORK}

Introducing robots as receptionists and/or welcoming agents in public spaces has been a topic of interest in multiple studies. The focus was on, among others, the robot's engagement strategies and its social behaviors. Pelachaud et al. [9] indicated that communicating the intent of a conversational agent (e.g., humanoid robot) is achieved through both verbal and non-verbal cues (e.g., [9]). In this section, we discuss the multi-modal design parameters to express the communication styles for social robots.

\section{A. Communication Styles}

Bales (1951), analyzed the interaction process between people and classified the communicative exchange into two areas namely the task area and the social-emotional area [10]. The focus of the task area is on executing and completing the current activity; while the social-emotional area focuses on expressing emotional states and reactions. From a sociolinguistic perspective [11], communication is centered on

\footnotetext{
${ }^{1}$ SoftBank Robotics, https: / / www. softbankrobotics.com
}

either the position (i.e., following predefined norms and rules) or the person (i.e., adapting the communication to the interlocutor). The focus of the position-centered (i.e., taskoriented) approach is on the task itself and the achievement of the required goals (e.g., [12], [13]); whereas the attention of the person-oriented communication (i.e., relationship oriented as in [14]) is more on the person with a focus on rapport building (e.g., [12], [13]).

\section{B. Design Parameters}

In the following we discuss the design parameters selected for expressing the task- and person-oriented communication styles.

Proxemic Behaviors: Michalowski et al. [15] proposed a spatial model of engagement after investigating the proxemic behaviors between visitors and Valerie, a receptionist robot. Similar research used proxemics for initiating an interaction from close distance (e.g., [16]) and for drawing people's attention from public distance (e.g., [17]). Furthermore, the four proxemic zones defined by Hall [18] were shown to be applicable in HRI (e.g., [19], as cited in [20]).

Voice: The quality of the voice was shown to have an impact on the interaction by drawing more attention and conveying emotional states (e.g., [21], [22]). McGinn and Torre [23] stressed on the importance of voice design in HRI and found that the robot voice should match its appearance. Trovato et al. [24] found that people preferred a receptionist agent to have a more anthropomorphic appearance with a human-like voice. Similarly, Niculescu et al. [25] found that the robot voice with high pitch (i.e., more animated) led to a better quality of interaction by increasing the robot's attractiveness and personal appeal.

Gestures and Gaze: Warm people are usually perceived as more sociable and less ruthless, which encourages initiating the interaction and eases the communication ([26], as cited in [27]). To evoke warmth-coldness when communicating with others, different behaviors can be used (e.g., [28]). Gestures (open versus close) and gaze (long versus short) are shown to evoke warmth-coldness (e.g., [29], [30], [31]).

Speech Acts: Speech acts play an important role in improving the quality and the efficiency of the interaction (e.g., [32], [33]). Zafari et al. [34] found that people enjoyed interacting with an informal robot that used motivational speech (i.e., was more person-oriented) when assisting them to perform a task. Similarly, Goetz et al. [35] indicated that a cheerful service robot was liked more than a serious robot. Sutherland et al. [36] also found that the robot friendliness (reflected via speech and smiles) had a positive effect on people's perception.

Eye Color: The use of colors was found to have an impact on manipulating human emotions by inducing feelings of relaxation or tension (e.g., [37], [38]). Terada et al. [39] validated the color-emotion relationship (i.e., anger-red, joy-yellow, trust-green, fear-violet, surprise-red and sadnessblue).

The aforementioned parameters were shown to affect people's interaction with social robots. In this study, these 
parameters will be combined and used, along with chit-chat, for designing multi-modal communicative robot behaviors that express the two communication styles, i.e., person- and task-oriented.

\section{Modeling The Communication Styles}

In this section, we will present the scenario of this study and discuss the modeling of the communication styles.

\section{A. Scenario}

When interacting with visitors at the reception desk, a receptionist is responsible for identifying their needs and providing them with the appropriate service. To play the role of an HCR assistant, a social robot needs to follow a sequence of actions or events (Fig. 2). The robot shall first initiate engagement (i.e., open the interaction) whenever a visitor is detected approaching it. Then, the robot shall offer its assistance. If it is able to assist the visitor, it will do so and give them the appropriate instructions; otherwise, it will refer them to the (human) HCR. Finally, the robot shall disengage (i.e., close the interaction) and wait for new visitors.

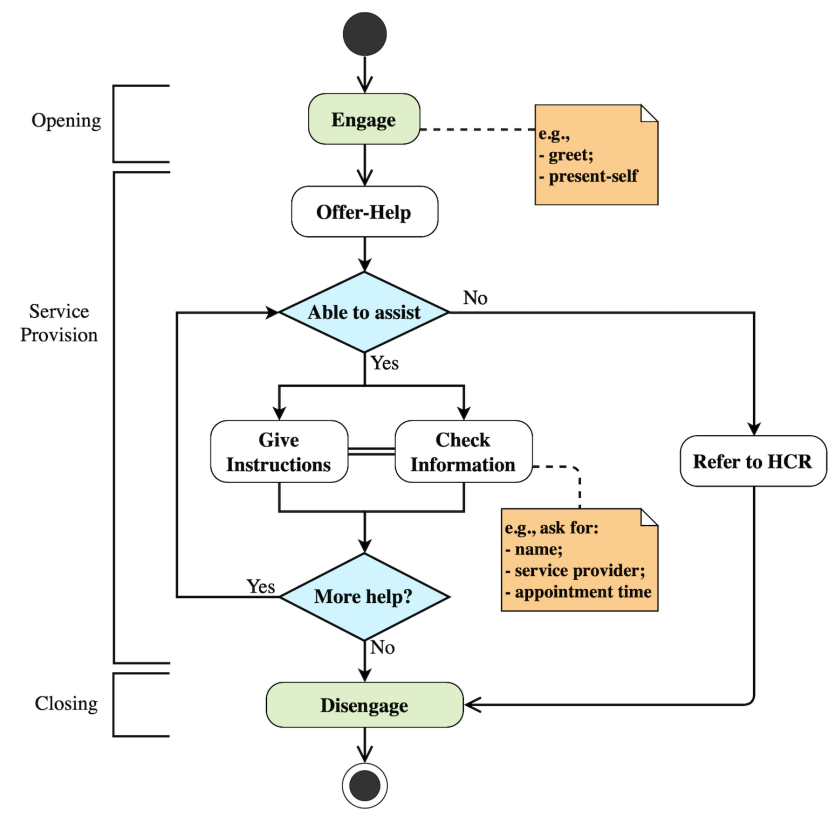

Fig. 2. Interaction flow of the reception task (explanation in text).

In this study, the communication styles of our robot will be designed using the case of a visitor entering a clinic to checkin for a scheduled appointment. Table II shows an example of a dialogue between the receptionist robot and a visitor.

\section{B. Design Blocks}

We separated the interaction into three stages (shown in Table I and Fig. 2) and created a list of blocks (as in [40]) for designing the interaction flow. The opening stage (i.e., initiate engagement) is composed of two blocks namely greet and present-self. The service-provision stage is composed of the blocks offer-help, check-information, confirm-information and give-instructions. Finally, the closing stage is composed of express-gratitude and farewell blocks. An additional chitchat block is also provided to give the designers the option to include chatting in the interaction.

TABLE I

SAMPle DiAlogue BETWEEN A ROBOT (Pepper) AND A Visitor (Kate) WANTING TO CHECK-IN FOR HER APPOINTMENT.

\begin{tabular}{l|ll}
\hline Stage & Speaker & Utterance \\
\hline Opening & Robot & $\begin{array}{l}\text { Good Morning. } \\
\text { My name is Pepper, I am here at your } \\
\text { service. }\end{array}$ \\
Visitor & $\begin{array}{l}\text { Hi Pepper. } \\
\text { Robot }\end{array}$ & How can I help you? \\
Visitor & I want to check-in for my appoint- \\
& ment. \\
Robot & And your name is? \\
Visitor & My name is Kate. \\
Robot & Welcome Kate. You have an appoint- \\
& ment with Dr. Smith at 10am, right? \\
& Visitor & Yes, that is correct. \\
Robot & Ok, you are checked-in. Please go to \\
& the waiting room and the doctor will \\
& be with you shortly. \\
& Thank you. \\
& Visitor & Have a nice day. \\
Robot & Bye. \\
\hline
\end{tabular}

\section{Parameters for Expressing the Styles}

To model the task-oriented and person-oriented communication styles, we derived a set of parameters (discussed in Section II-B. The settings of the parameters can be adjusted for expressing a given style.

As shown in Table II the gesture openness is reflected via a list of open gestures (i.e., for a more inviting and warmer communication) and close gestures (i.e., for a more distant and colder communication). The robot's gaze can be set to either fixated on the communicative partner or diverted. The distance between the robot and the user (i.e., the proxemic closeness) can also be adjusted from close (.5m or less) to far (beyond $2 m$ ). The robot voice is manipulated via the pitch, from low at $50 \mathrm{~Hz}$ to high at $200 \mathrm{~Hz}$ (default setting at $100 \mathrm{~Hz}$ ); the speed (i.e., speech rate in words per minute), from slow at 50wpm to fast at $300 \mathrm{wpm}$ (default setting at $100 \mathrm{wpm}$ ); and the prosody, either weak or strong. The speech acts are varied to express a formal or an informal communication. A chit-chat option is also added for rapport building. Finally, the color of the robot's eyes can be set to white (neutral), red, green or blue.

\section{TABLE II}

DESIGN PARAMETERS FOR EXPRESSING THE COMMUNICATION STYLES

\begin{tabular}{|c|c|c|}
\hline \multicolumn{2}{|c|}{ Parameter } & Variation \\
\hline \multirow{3}{*}{\multicolumn{2}{|c|}{$\begin{array}{l}\text { Gesture Openness } \\
\text { Gaze Diversion } \\
\text { Proxemic Closeness (m) }\end{array}$}} & Close or Open \\
\hline & & Fixated or Diverted \\
\hline & & from Close to Far \\
\hline \multirow{3}{*}{ Voice } & Pitch $(\mathrm{Hz})$ & from Low to High \\
\hline & Speed (wpm) & from Slow to Fast \\
\hline & Prosody & Weak or Strong \\
\hline \multicolumn{2}{|c|}{ Speech Acts } & Informal or Formal \\
\hline \multicolumn{2}{|c|}{ Chit-Chat } & No-Chat or Chat \\
\hline \multicolumn{2}{|c|}{ Eye Color } & White / Red / Green / Blue \\
\hline
\end{tabular}




\section{EVALUATION}

First, to evaluate the designs for expressing the taskoriented and person-oriented communication styles, we formulated the following hypothesis:

H1: Task- and person-oriented communication styles are designed differently for social robots as predicted by theory. Differences will be measured in terms of the design parameters used in the styles.

Second, to evaluate the design method, we formulated the following two hypotheses:

H2: The iterative design converges. Convergence is a gradual decline of the distance in parameters between the design at each iteration and the final iterative design.

H3: The individual designs are incorporated in the iterative design. Incorporation is measured by the influence of the individual designs on the parameter-settings of the iterative design.

On top of these hypotheses, we also evaluated the usability and reception of our design method and tool using a questionnaire.

\section{Design Method}

To test our hypotheses, we conducted an experiment for designing the communication styles of a robot playing the role of an HCR assistant. The experimental methods and procedures were approved by the human research ethics committee of the university.

\section{A. Participants}

The participants $(N=13)$ were university graduate students recruited (by advertisements) from the department of Industrial Design Engineering. They consisted of 5 males and 8 females with an average age of 23.23 years $(S D=1.88)$. All participants mentioned (via a questionnaire) that they had intermediate experience in design with no experience in human-robot interaction. As a compensation for their time, each participant received a 10 EURO voucher after completing the experiment.

\section{B. Materials}

To design the robot communication styles, we provided the participants with the necessary materials and tools.

1) Robotic Platform: In this study, we used a humanoid robot, Pepper 1 (Fig. 1), as the robotic platform for testing the communication styles. We setup Pepper to autonomously engage users, i.e., initiate and maintain engagement.

2) Design Tool: We developed an interaction-design (ID) prototyping tool (Fig. 33 to design the communication styles and deploy them directly on the Pepper robot. A refined version of the $I D$-tool is available for download 2 Via the tool, the designers (i.e., participants) were able to construct an interactive dialogue for each style using a set of design blocks (Section III-B). The blocks (Fig. 3.B) were separated into three communication stages namely opening, serviceprovision and closing. To design the styles, users could drag

${ }^{2}$ Interaction-Design Tool for Multi-Modal Communication, https:// github.com/ES-TUDelft/robot-interaction-tool

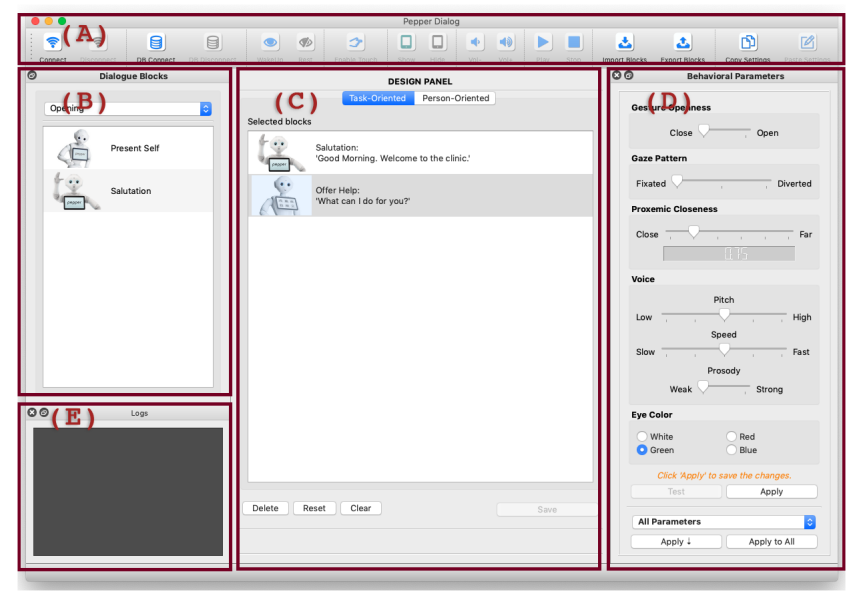

Fig. 3. The user Interface for designing the communication styles and testing them on the robot. (A) top menu bar; (B) dialogue blocks separated into communication stages; (C) design panel for creating the interaction flow; (D) parameters to be tuned for each block; (E) log panel.

and drop blocks to the design panel (Fig. 3.C) and create an interaction flow. The parameter-settings of the blocks could be adjusted for expressing each communication style by means of sliders, buttons or item selection (Fig. 3D). The adjustments could be immediately tested on Pepper by selecting one block (i.e., clicking the test button) or playing the complete scenario (i.e., clicking the play button). Pepper would autonomously initiate engagement (i.e., when the user is detected in its engagement zone) and execute the designed interaction flow.

3) Questionnaire: We used a questionnaire for the participants to indicate, on a 7-point Likert scale (higher=better), the usability of the system (i.e., ease of use, ease of learning and satisfaction) and their satisfaction after completing the scenario. The questionnaire was based on the USE Questionnaire by Lund [41] (with the exclusion of the system usefulness score for not being applicable in our case) and the after-scenario questionnaire by Lewis [42]. Two additional questions were introduced to assess the participants' confidence about their design and the distinctiveness of the styles.

\section{Experimental Design}

In this study, we manipulated the design task based on the intended communication style (i.e., independent variable) of a social robot. Each subject was asked to design both a person-oriented and a task-oriented version of a hospital reception robot.

1) Interaction Design: To design the styles, we adopted an iterative interaction-design (ID) method. The $I D$-method distinguishes the creation of an individual design and the contribution to the iterative design. In the individual step, the designer would provide his/her individual design of each communication style (i.e., task and person-oriented) by adjusting the parameter-settings with no influence from other designs. In the second iterative step, the designer would adjust the parameter-settings of two iterative de- 
signs (i.e., produced by the previous designers) namely one person-oriented design $(P O D)$, and one task-oriented design $(T O D)$. As illustrated in Fig. 4, each iteration Iter $(i)$ consisted of adjusting the previous designs, i.e., $P O D(i-1)$ and $T O D(i-1)$, in order to fine-tune the parameters and produce $P O D(i)$ and $T O D(i)$.

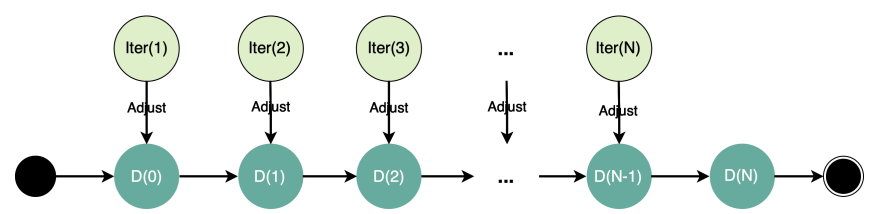

Fig. 4. Illustration of the iterative step from the design method. In each iteration $i$ of $N$, the previous design $D(i-1)$ is adjusted to produce a new design $D(i)$, with $i$ going from 1 to $N$.

For the iterative step, we prepared two initial designs, $P O D(0)$ and $T O D(0)$, and set the parameters to reflect the styles (based on the literature and our own judgement). The person-oriented style was modeled to be warm and open with informal messages; open gestures; a fixated gaze pattern; close proxemics (distance set to $.5 \mathrm{~m}$ ); green eye color; and a voice with high pitch, strong prosody and slow pace. The task-oriented style was modeled to be more distant and direct with formal messages; close gestures; a diverted gaze pattern; proxemics set to $1 \mathrm{~m}$; neutral (white) eye color; and a voice with low pitch, weak prosody and fast pace.

In the end, each subject thus delivered 4 different designs: two person-oriented designs (an individual one and the next iterative design) and two task-oriented designs (an individual one and the next iterative design).

2) Measurements: During the experiment, we collected data to analyze different measures (i.e., dependent variables) for verifying our hypotheses.

a) Design parameters: We recorded the settings of the parameters for expressing the styles (Section II-B. Further, we recorded the use of the chit-chat interaction blocks when designing the styles. We also recorded the total number of blocks to design each style. Finally, we recorded the time spent interacting with the robot when executing the reception task following the designed styles. These measures will be useful for comparing the styles $(H 1)$.

b) Euclidean Distances: Using the parameter-settings obtained from designing the styles, we computed the euclidean distance between the final iterative design (i.e., at iteration $N$ ) and the designs from both the individual and iterative steps. This will be used for verifying $H 2$ (design convergence). In addition, we computed the difference in euclidean distances $(\Delta d)$ between the iterative designs (at iterations $i-1$ and $i$ ) and the individual design (at iteration $i$ ) as in (1). This measure will be used to analyze the personal influence on the iterative design (H3). If $\Delta d$ is negative, i.e., the distance between the individual design and the next iterative design becomes smaller, this is interpreted as an influence of the individual design on the iterative design.

$$
\Delta d=\operatorname{dist}\left(\operatorname{Iter} D_{i}, \operatorname{Ind} D_{i}\right)-\operatorname{dist}\left(\operatorname{Iter} D_{i-1}, \operatorname{Ind} D_{i}\right)
$$

where dist is the Euclidean distance; Iter $D$ and $I n d D$ are the iterative and individual designs; and $i$ is the iteration number.

\section{Procedure}

The study took place in a quiet room and consisted of a one-to-one session between each participant and the robot Pepper, with the presence of one researcher. The participants sat on a chair behind a workstation with Pepper on the side. To interact with Pepper (i.e., when a design is deployed on the robot), they had to stand up and move toward it. Once Pepper detected a participant entering its engagement zone (within $2 \mathrm{~m}$ ), it would autonomously initiate the engagement and execute the interactive dialogue.

Prior to data collection, the participants were given an explanation about the purpose of the study, the communication styles and the tasks required to complete the experiment (verbally and via an information sheet). The participants were also provided with a user interface (i.e., the $I D$-tool) to design the styles by creating an interaction with Pepper using a set of design blocks (as in Section V-B.2). Via the tool, they could modify the blocks' parameters to express the styles.

After signing the consent form, the participants tested the design environment by creating a trial interaction with Pepper using three to four design blocks with random parametersettings. The aim of the trial was to familiarize them with the $I D$-tool interface and the effect of the parameters on the robot's expressiveness. Then, the participants could proceed with the design experiment. Firstly, they would create their own designs of the communication styles (i.e., task versus person). Secondly, they would validate two iterative designs (one for each style) and adjust the parameters, if necessary.

Finally, the participants were asked to fill-in a questionnaire about the usability of the design method and tool. The experiment took less than an hour and a half (Mean = 75.62 min, $S D=14.74 \mathrm{~min}$ ) and the starting style design of the test conditions (task versus person) was done in a counterbalanced fashion.

\section{Results AND ANALYSis}

In total we obtained 13 times 4 designs. In this Section we will analyze the designs and verify our hypotheses.
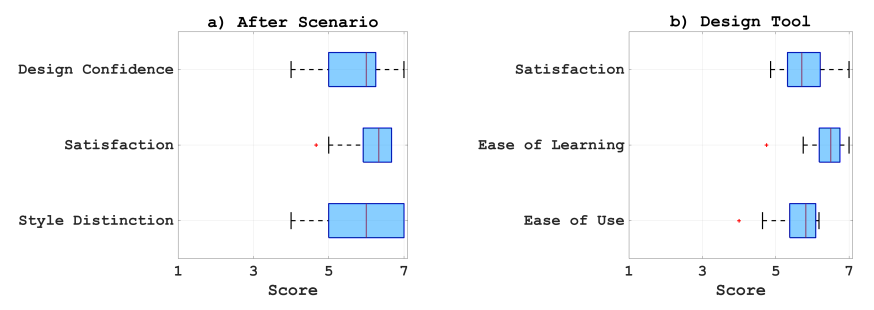

Fig. 5. Distribution of the questionnaire scores for the after-scenario (a) and tool usability (b).

\section{A. Design Environment}

The questionnaire results (Fig. 5) show that the participants were satisfied by the scenario and task completion 
(Mean $=6.10, S D=0.79)$, were confident of their designs $($ Mean $=5.62, S D=1.04)$ and were able to distinguish between the styles (Mean $=6.0, S D=1.0$ ). The participants also provided satisfactory ratings for the $I D$-tool as easy to use (Mean $=5.59, S D=1.29$ ), easy to learn (Mean $=6.35, S D=0.79$ ) and satisfactory $($ Mean $=5.78, S D=1.33)$.

\section{B. Block Usage for Expressing the Styles}

The number of blocks per design (Fig. 6a) was significantly lower for the task- than the person-oriented in both the individual and iterative designs (one-way ANOVA, $F(1,24)=18.05, p<.001$; and $F(1,24)=21.0, p<$ .001 , respectively). Furthermore, the interaction time (Fig. 6 b) was significantly shorter for the task- than the personoriented style in the individual and iterative designs (oneway ANOVA, $F(1,24)=12.53, p<.001$; and $F(1,24)=$ $70.27, p<.001$, respectively). These findings support $H 1$, i.e., that a task-oriented style is designed differently and is distinguishable from a person-oriented style on a high level.
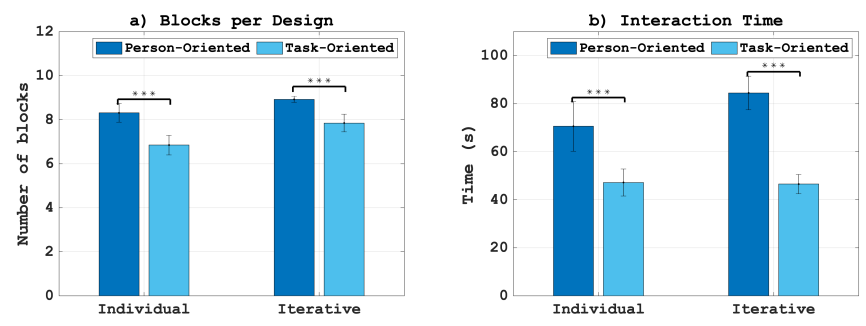

Fig. 6. Mean measurements (with error bars showing 95\% CI) for the a) number of blocks per design; and $b)$ interaction time. $(* * *=$ significant at $.001)$

\section{Parameter-Settings for Expressing the Styles}

The gestures for the person-oriented style were designed to be open more often than closed compared to the taskoriented style (Fig. 7 a-b). This was significant only for the iterative designs (Fisher's exact test, $p<.001$ ).

The gaze diversion (Fig. 77.c-d) showed no significant difference between the styles (Fisher's exact test, $p>.05$ ).

The speech acts (Fig. 7]e-f) were significantly more formal for the task- than the person-oriented for both the individual and iterative designs (Fisher's exact test, $p<.05$ and $p<$ .001, respectively). Similarly, the use of chit-chat (Fig. 7.g-h) was significantly lower for the task- than the person-oriented in the individual and iterative designs (Fisher's exact test, $p<.01$ and $p<.001$, respectively).

The prosody settings (Fig. 7 , i-j) showed no meaningful difference between the styles.

Eye-colors (Fig. 7]k-1) were significantly different between the individual designs but not the iterative designs $\left(\chi^{2}=\right.$ $9.60, p<.05$; and $\chi^{2}=1.88, p>.05$, respectively). Green was most preferred for the person-oriented style; red was never used in both styles.

The proxemic settings (Fig. 8.a) were within the personal zone (less than $1.2 \mathrm{~m}$, as defined by Hall [18]) with no significant difference between the styles in both the individual

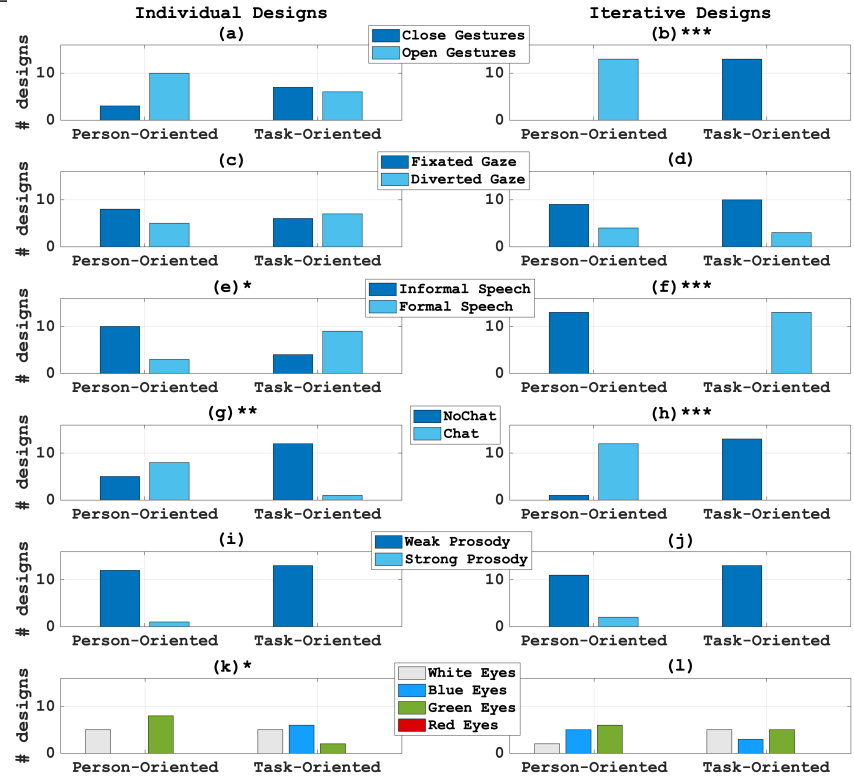

Fig. 7. Variations in the parameter-settings for the gestures $(a, b)$; gaze (c, d); speech acts (e, f); use of chit-chat $(\mathrm{g}, \mathrm{h})$; prosody $(\mathrm{i}, \mathrm{j})$; and eye color $(\mathrm{k}, \mathrm{l})$, for the individual and iterative designs. $(*=$ significant at $.05 ; * *=$ significant at $.01 ; * * *=$ significant at .001$)$

and iterative designs (one-way ANOVA, $F(1,24)=2.01$, $p>.05$; and $F(1,24)=0.65$, ns, respectively).

For the voice settings, the pitch (Fig. 8b) showed no significant differences (one-way ANOVA, $F(1,24)<1$, ns). The speech rate (Fig. 8.c) was within the normal range and the difference between the styles for the iterative designs was small but significant (one-way ANOVA, $F(1,24)=10.49$, $p<.05)$. The task-oriented style was (slightly) faster in the iterative design.
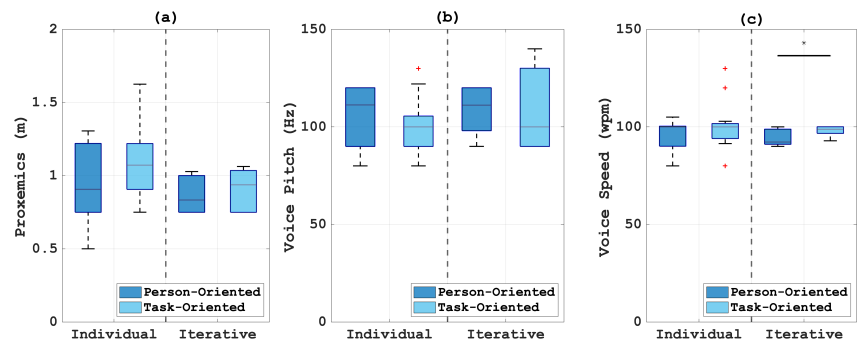

Fig. 8. Variations in the settings for the a) proxemics; b) voice pitch; and c) speech rate. $(*=$ significant at .05$)$

The aforementioned findings from the parameter-settings imply the presence of design variations in expressing the styles, which support $H 1$. The variations were more evident in the iterative than in the individual designs.

\section{Design Method}

To compare the individual and iterative steps of the $I D$ method, we computed the Euclidean distances between the final iterative design and the designs obtained from both steps (Section V-C.2.b. The individual designs (Fig. 9. Left) appear to be more influenced by the designers' preferences 
(i.e., are more personalized) than the iterative designs (Fig. 9-Right). They (i.e., the individual) show a more erratic process compared to the iterative design, which show a more stable and convergent process. This is an indication of the convergence of the iterative design, which supports $H 2$.
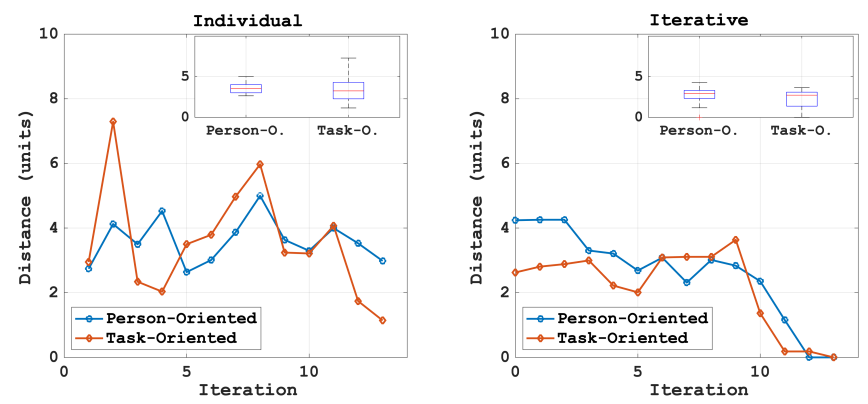

Fig. 9. Euclidean distances between the final iterative design and the designs from the individual and iterative steps of the design method.

Convergence is also an indication that, in the iterative step, the designers reflected on what they did in the individual step and used this to refine the iterative design rather than change it drastically to fit their own ideas. To proof this, we quantified the personal influence on the parameter-settings of the iterative designs (as discussed in Section V-C.2.b and illustrated in Fig. 10). Negative values indicate an influence of the individual design on the iterative design. The $\Delta d$ was significantly different from 0 in the designs expressing the person-oriented style $(t(12)=-2.76, p<.05)$ but not the task-oriented style $(t(12)=-1.64, p>.05)$. This implies that the person-oriented iterative designs were significantly influenced by the individual designs. These findings support H3.

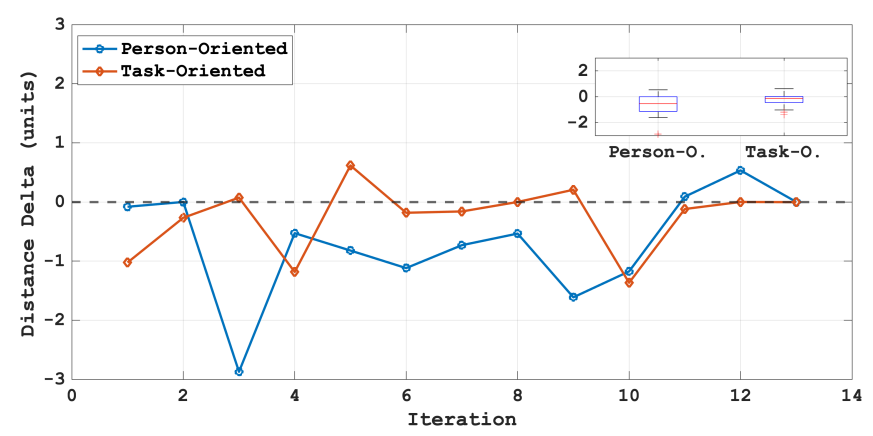

Fig. 10. Difference in the euclidean distances $(\Delta d)$ between the iterative designs (at iterations $i$ and $i-1$ ) and the individual design (at $i$ ). This is a measure of the influence of the individual design of each participant on the iterative design (for both styles). Negative numbers indicate that the iterative design is moving in the direction of the individual design.

\section{Discussion AND CONCLUSION}

This paper presented an iterative development method (i.e., the $I D$-method), in which a designer first creates his/her "own" individual design and, subsequently, provides an iteration to the evolving joint design. We developed an $I D$-tool and evaluated its usage with designers. The tool provides support to the iterative creation of a design by (a) selecting the theory-based communication style, (b) creating and linking the dialogue act components for the concerning use case, and (c) setting the associated expression parameters.

The $I D$-method and tool proved to be effective for designing multi-modal communicative behaviors for social robots, i.e., the dialogue acts with the desired parameter settings. It is worth to mention that whenever a design distinction between the styles was found in the individual designs, it appeared more evident in the iterative design (for example, the gesture openness and speech acts in Fig. 7). Overall, the designs converged and individual designs were harmonized into the iterative design. This indicates that the designers were able to reflect on their own individual designs when tuning the iterative design towards a stable end point.

The process and outcome of the design of a task- or a person-oriented interaction, proved to differ for the two communication styles. The task-oriented style showed a more formal, shorter and less chatty communication. Both the individual and iterative designs showed that the personoriented style led to a significantly longer interaction time with the Pepper robot. This was further corroborated through the significant use of chit-chat and the higher number of blocks for expressing the person- versus the task-oriented style. It seems that a robot with a person-oriented style is expected to spend more time interacting with visitors. This finding is consistent with [34] who found that people tend to engage longer with a robot that gives more personalized and motivational feedback, i.e., is more person-oriented.

Furthermore, the findings show that a person-oriented style is reflected through informal speech acts and open gestures compared to the task-oriented style. These findings are consistent with [30] which showed that open gestures express more warm and welcoming interaction (i.e., more person-oriented) whereas close gestures express more cold and distant interaction (i.e., more task-oriented). They are also consistent with [34] where a robot was perceived as informal and more agreeable when it provided personalized feedback than when it adopted a task based approach.

The distance between the robot and visitors was set within the personal zone for both styles. There was also no significant nor relevant variations in the voice settings between the styles. These findings show a consistency in the voice design for the Pepper robot, which is inline with [23] who indicated the importance of a match between both the robot voice and its appearance. It is worth to mention that the voice pitch was not used to make the robot more attractive as in [25], which may be due to the serious setting related to healthcare.

In conclusion, the design method and tool provided the desired theory-based multi-modal communicative behaviors, validating its usefulness. Future work will validate, via an empirical study, the communication styles for this application domain of a reception robot and, subsequently, extract reusable design patterns, cf. [40]. For the selected design problem, the $I D$-tool had a satisfactory usability. This tool is being improved to support the creation of more complex interaction designs. 


\section{REFERENCES}

[1] Y. Kato, T. Kanda, and H. Ishiguro, "May i help you? - design of human-like polite approaching behavior-," in ACM/IEEE International Conference on Human-Robot Interaction (HRI), Mar 2015, pp. 35-42.

[2] K. Mizumaru, S. Satake, T. Kanda, and T. Ono, "Stop doing it! approaching strategy for a robot to admonish pedestrians," in ACM/IEEE International Conference on Human-Robot Interaction (HRI), Mar 2019, pp. 449-457.

[3] P. M. Neuwelt, R. A. Kearns, and I. R. Cairns, "The care work of general practice receptionists," Journal of primary health care, vol. 8, pp. 122-129, June 2016.

[4] H. Hewitt, L. McCloughan, and B. McKinstry, "Front desk talk: discourse analysis of receptionist-patient interaction," British Journal of General Practice, vol. 59, no. 565, pp. e260-e266, 2009.

[5] J. Ward and R. Mcmurray, "The unspoken work of general practitioner receptionists: a re-examination of emotion management in primary care," Social science \& medicine, vol. 72, pp. 1583-7, March 2011.

[6] S. Arber and L. Sawyer, "The role of the receptionist in general practice: A 'dragon behind the desk'?" Social Science \& Medicine, vol. 20, no. 9, pp. $911-921,1985$.

[7] M. Alazri, P. Heywood, and B. Leese, "How do receptionists view continuity of care and access in general practice?" European Journal of General Practice, vol. 13, no. 2, pp. 75-82, 2007.

[8] J. Xu, J. Broekens, K. Hindriks, and M. A. Neerincx, "Mood expression through parameterized functional behavior of robots," in 2013 IEEE RO-MAN, Aug 2013, pp. 533-540.

[9] C. Pelachaud, N. I. Badler, and M. Steedman, "Generating facial expressions for speech," Cognitive Science, vol. 20, pp. 1-46, 1996.

[10] R. F. Bales, Interaction Process Analysis: A method for the study of small groups. Cambridge, Massachusetts: Addison-Wesley Press Inc., 1951, ch. 2: Theoretical framework, pp. 30-84.

[11] K. Miller, Communication Theories: Perspectives, Processes and Contexts, 2nd ed. New York: McGraw Hill, 2005.

[12] C. Froehle, "Service personnel, technology, and their interaction in influencing customer satisfaction," Decision Sciences - DECISION SCI, vol. 37, February 2006.

[13] I. Kang, S. Han, and J. Lee, "Task-oriented and relationship-building communications between air traffic controllers and pilots," Sustainability, vol. 9, no. 10, 2017.

[14] J. D. Pincus, "Communication satisfaction, job satisfaction and job performance," Human Communication Research, vol. 12, no. 3, pp. 395-419, 1986.

[15] M. P. Michalowski, S. Sabanovic, and R. Simmons, "A spatial model of engagement for a social robot," in 9th IEEE International Workshop on Advanced Motion Control, 2006., March 2006, pp. 762-767.

[16] P. Holthaus, K. Pitsch, and S. Wachsmuth, "How can i help?" Int. Journal of Social Robotics, vol. 3, no. 4, pp. 383-393, 2011.

[17] E. Saad, J. Broekens, M. A. Neerincx, and K. V. Hindriks, "Enthusiastic robots make better contact," in IEEE/RSJ International Conference on Intelligent Robots and Systems (IROS), Macau, China, Nov 2019, pp. 1094-1100.

[18] E. T. Hall, The Hidden Dimension. Garden City, N.Y.: Doubleday, 1966, ch. X: Distances in Man, pp. 113-129.

[19] J. Mumm and B. Mutlu, "Human-robot proxemics: Physical and psychological distancing in human-robot interaction," in ACM/IEEE International Conference on Human-Robot Interaction (HRI), March 2011, pp. 331-338.

[20] D. G. Macharet and D. A. Florencio, "Learning how to increase the chance of human-robot engagement," in IEEE/RSJ International Conference on Intelligent Robots and Systems, Nov 2013, pp. 21732179.

[21] T. Otsuka, K. Nakadai, T. Takahashi, K. Komatani, T. Ogata, and H. G. Okuno, "Voice-awareness control for a humanoid robot consistent with its body posture and movements," Paladyn, vol. 1, no. 1, pp. 80-88, Mar 2010.

[22] M. Schwenk and K. O. Arras, "R2-d2 reloaded: A flexible sound synthesis system for sonic human-robot interaction design," in The 23rd IEEE International Symposium on Robot and Human Interactive Communication, Aug 2014, pp. 161-167.

[23] C. McGinn and I. Torre, "Can you tell the robot by the voice? an exploratory study on the role of voice in the perception of robots," in 2019 14th ACM/IEEE International Conference on Human-Robot Interaction (HRI), March 2019, pp. 211-221.
[24] G. Trovato, J. G. Ramos, H. Azevedo, A. Moroni, S. Magossi, H. Ishii, R. Simmons, and A. Takanishi, "Designing a receptionist robot: Effect of voice and appearance on anthropomorphism," in IEEE RO-MAN, Aug 2015, pp. 235-240.

[25] A. Niculescu, B. Van Dijk, A. Nijholt, H. Li, and S. L. See, "Making social robots more attractive: The effects of voice pitch, humor and empathy," International Journal of Social Robotics, vol. 5, no. 2, pp. 171-191, Apr 2013.

[26] W. N. Widmeyer and J. Loy, "When you're hot, you're hot! warmcold effects in first impressions of persons and teaching effectiveness," Journal of Educational Psychology, vol. 80, pp. 118-121, 031988.

[27] R. L. Boyd, K. Bresin, S. Ode, and M. D. Robinson, "Cognitive egocentrism differentiates warm and cold people," Journal of research in personality, vol. 41, no. 7, pp. 90-96, Feb. 2013.

[28] R. Peters, J. Broekens, and M. A. Neerincx, "Robots educate in style: The effect of context and non-verbal behaviour on children's perceptions of warmth and competence," in 2017 26th IEEE International Symposium on Robot and Human Interactive Communication (ROMAN), Aug 2017, pp. 449-455.

[29] A. Kendon and M. Cook, "The consistency of gaze patterns in social interaction," British journal of psychology (London, England : 1953), vol. 60, pp. 481-94, December 1969.

[30] T.-H. D. Nguyen, E. Carstensdottir, N. Ngo, M. S. El-Nasr, M. Gray, D. Isaacowitz, and D. Desteno, "Modeling warmth and competence in virtual characters," in Intelligent Virtual Agents, W.-P. Brinkman, J. Broekens, and D. Heylen, Eds. Cham: Springer International Publishing, 2015, pp. 167-180.

[31] P. Prajod, M. Al Owayyed, T. Rietveld, J.-J. van der Steeg, and J. Broekens, "The effect of virtual agent warmth on human-agent negotiation," in Proceedings of the 18th International Conference on Autonomous Agents and MultiAgent Systems, ser. AAMAS '19. Richland, SC: International Foundation for Autonomous Agents and Multiagent Systems, 2019, pp. 71-76.

[32] J. Cassell, T. Bickmore, L. Campbell, H. Vilhjalmsson, and H. Yan, "Conversation as a system framework: Designing embodied conversational agents," 2000.

[33] B. Granström and D. House, "Effective interaction with talking animated agents in dialogue systems," in Advances in Natural Multimodal Dialogue Systems. Springer Netherlands, 2005, pp. 215-243.

[34] S. Zafari, I. Schwaninger, M. Hirschmanner, C. Schmidbauer, A. Weiss, and S. T. Koeszegi, "you are doing so great! the effect of a robot's interaction style on self-efficacy in hri," in $201928 \mathrm{th}$ IEEE International Conference on Robot and Human Interactive Communication (RO-MAN), Oct 2019, pp. 1-7.

[35] J. Goetz and S. Kiesler, "Cooperation with a robotic assistant," in Extended Abstracts on Human Factors in Computing Systems, ser. CHI EA '02. New York, NY, USA: ACM, 2002, pp. 578-579.

[36] C. J. Sutherland, B. K. Ahn, B. Brown, J. Lim, D. L. Johanson, E. Broadbent, B. A. MacDonald, and H. S. Ahn, "The doctor will see you now: Could a robot be a medical receptionist?" in 2019 International Conference on Robotics and Automation (ICRA), May 2019, pp. 4310-4316.

[37] V. Patricia and M. Albert, "Effects of color on emotions," Journal of Experimental Psychology, vol. 123, no. 4, pp. 394-409, 1994.

[38] S. Wang and R. Ding, "A qualitative and quantitative study of color emotion using valence-arousal," Frontiers of Computer Science, vol. 6, no. 4, pp. 469-476, Aug 2012.

[39] K. Terada, A. Yamauchi, and A. Ito, "Artificial emotion expression for a robot by dynamic color change," in 2012 IEEE RO-MAN: The 21st IEEE International Symposium on Robot and Human Interactive Communication, Sep. 2012, pp. 314-321.

[40] A. Sauppé and B. Mutlu, "Design patterns for exploring and prototyping human-robot interactions," in Proceedings of the SIGCHI Conference on Human Factors in Computing Systems. New York, NY, USA: Association for Computing Machinery, 2014, p. 14391448.

[41] A. Lund, "Measuring usability with the use questionnaire," Usability and User Experience Newsletter of the STC Usability SIG, vol. 8, 01 2001.

[42] J. R. Lewis, "Ibm computer usability satisfaction questionnaires: Psychometric evaluation and instructions for use," International Journal of Human-Computer Interaction, pp. 57-78, 1995. 